\title{
Relationship between mechanical properties and age-hardening behavior around grain boundaries of Al-Mg-Si alloy
}

\author{
Takuya Hashimoto $^{1, *}$, Ken-ichi $\mathrm{Ikeda}^{2}$, and Seiji Miura ${ }^{2}$ \\ ${ }^{1}$ Graduate School of Engineering, Hokkaido University, Kita13, Nishi8, Kita-ku, Sapporo, Hokkaido, 060-8628, Japan \\ ${ }^{2}$ Faculty of Engineering, Hokkaido University, Kita13, Nishi8, Kita-ku, Sapporo, Hokkaido, 060-8628, Japan
}

\begin{abstract}
Nanoindentation tests were conducted near the grain boundary (GB) of the Al-Mg-Si alloy, and the influence of GB character on the aging precipitation behavior and the mechanical properties was confirmed. After obtaining the GB characters by electron back scattered diffraction (EBSD) analysis, nanoindentation tests were carried out on under-aged, peak-aged, and over-aged samples. And then, the indentation areas were observed by back scattered electrons imaging (BSE) in order to identify indentation positions with respect to the GB. In this study, for the GB character, focusing on the rotation angle, the highangle GB (HAGB) and the low-angle GB (LAGB) were selected. In addition, coincident site lattice GBs (CSL) were selected as the special GB. In the $180^{\circ} \mathrm{C}$ under-aged samples, the nano-hardness near GB is higher than that far from $\mathrm{GB}$, while $180^{\circ} \mathrm{C}$ peak-aged samples, the nano-hardness is lower than that far from GB. Then the range near the GB where the hardness changes was larger at HAGB than at LAGB and CSL3. This suggests that the GB character affects the aging precipitation behavior and mechanical properties.
\end{abstract}

\section{Introduction}

Al-Mg-Si alloys are superior in workability and corrosion resistance as well as bake hardenability (age hardenability) compared to other aluminum alloys and are used as body panels for automobiles. Bake hardenability means that the hardness increases by heat treatment of paint bake after molding the body of an automobile, and it is considered that the expression of this hardenability is attributed to aging precipitates formed during heat treatment. Therefore, when developing products utilizing the age-hardening properties of Al-Mg-Si alloys, it is necessary to understand the effects of aging precipitates on strength.

When an aging heat treatment is applied to an Al-Mg$\mathrm{Si}$ alloy, it is proposed that the aging precipitates transition in the following process [1-2]:

$$
\mathrm{SSSS} \rightarrow \mathrm{Mg}-\mathrm{Si} \text { cluster } \rightarrow \text { GP zone } \rightarrow \beta^{\prime \prime} \rightarrow \beta^{\prime} \rightarrow \beta .
$$

Precipitates that are predominantly precipitated in the alloy differ depending on the aging temperature and time, and $\beta^{\prime \prime}$ is a precipitate involved in the automobiles body manufacturing process.

As the heat treatment is continued, $\beta^{\prime \prime}$ eventually transits to $\beta^{\prime}$, a rod-like semi-coherent precipitate, and to plate-like non-coherent precipitate, $\beta\left(\mathrm{Mg}_{2} \mathrm{Si}\right)$, the final stable phase. They have a smaller contribution to strength as compared to $\beta^{\prime \prime}$ and are rarely used as the main reinforcing phase. However, $\beta^{\prime}$ and $\beta$ themselves are considered to precipitate on the grain boundaries depending on the heat treatment temperature and time. In order to clarify the intergranular fracture during the tensile tests and the effect of GB precipitates on the improvement of alloy strength, it is necessary to clarify the characteristics of the GB precipitates. Therefore, it is necessary to clarify the nature of GB precipitates.

As the strength within each grain interior of the polycrystalline material increases, embrittlement due to GB fracture occurs, and the effect of the GB cannot be ignored when using structural materials. Matsuda et al. [3] reported the relationship between the Schmid factor for the tensile axis and the stress transfer coefficient in the age-hardened Al-Mg-Si-based alloy, which is expanding its application as a lightweight structural material. Cai et al. [4] also systematically classified the precipitate-free zone (PFZ), which is one of the factors preventing the increase in strength, in the age hardening type Al-Zn-Mg$\mathrm{Cu}$ alloy, which has the highest strength among aluminum alloys, based on GB character. The width of the PFZ tends to be wider as the quenching speed decreases [5], which reduces the strength of the material. In order to reduce the PFZ width, it is necessary to narrow the PFZ width by increasing the quenching rate.

Here, the GB character is the property of the GB classified by the relative orientation (rotation angle / axis) of the adjacent crystal grains and the grain interface orientation, and this property is known to greatly affect various properties of a material. Random GBs, which are ordinary GBs, have a high energy structure and are likely to cause GB degradation phenomena. On the other hand, there is a grain boundary with a regular arrangement of atoms and few gaps, called the corresponding grain boundary. On the other hand, there is a type of grain

Corresponding author: takuya_hashimoto@eis.hokudai.ac.jp 
Table 1

Chemical compositions of the alloys utilized in this work (mass\%).

\begin{tabular}{cccccccc||c||c}
\hline $\mathrm{Al}$ & $\mathrm{Si}$ & $\mathrm{Fe}$ & $\mathrm{Mn}$ & $\mathrm{Mg}$ & $\mathrm{Zn}$ & $\mathrm{Cr}$ & $\mathrm{Ti}$ & $\mathrm{Mg} / \mathrm{Si}$ \\
\hline Bal. & 0.344 & 0.042 & 0.001 & 0.621 & 0.003 & 0.001 & 0.011 & 2.1 \\
\hline
\end{tabular}

boundary called the coincidence site lattice boundary (CSL GB), in which atoms are regularly arranged with a small number of gaps between them. In particular, the $\Sigma 3$ GB, which is classified as CSL GB, has low GB energy. It is also considered to be less prone to GB degradation phenomena in aluminium [6]. Therefore, GB engineering (GBE), which is a technique intended to improve the characteristics of polycrystalline materials by introducing such corresponding grain boundaries into many materials, has been developed [7]. As an application example Shimada et al. [8] reported that in SUS304L, random GBs that could be the starting point of corrosion and fracture were reduced, some CSL GBs were introduced, and the corrosion rate was suppressed to one-quarter of that of the base metal. As mentioned above, attention has been paid to studies relating the properties of materials and the factors affecting them to the GB character. However, at present, there are many points to be discussed about the influence of the GB character on the mechanical properties through the aging precipitation process.

In this study, Al-Mg-Si based alloys subjected to under-aging, and peak-aging heat treatments and pure aluminum from which $\mathrm{Mg}$ and $\mathrm{Si}$ were removed from the Al alloy were subjected to nanoindentation test and transmission electron microscopy (TEM) observation of near GBs. And the influence of GB character on aging precipitation behavior and mechanical properties was confirmed.

\section{Experimental procedure}

\subsection{Under-aged heat treatment}

The chemical compositions of the alloys used in this study are listed in Table 1. The forged material was homogenized at $530^{\circ} \mathrm{C}$ for 6 hours, and then hot-rolled to a thickness of $4 \mathrm{~mm}$. After that, a sample that had been subjected to cold rolling to have a thickness of $4 \mathrm{~mm}$ to 1 mm was used as a sample and was cut so that $\mathrm{RD} \times \mathrm{TD}$ was $15 \mathrm{~mm} \times 10 \mathrm{~mm}$ to obtain a sample.

The samples were solution treated at $550^{\circ} \mathrm{C}$ for 1 hour and subsequently quenched into iced water to produce a supersaturated solid solution (SSSS). Then, the average grain size was about $350 \mu \mathrm{m}$. Thereafter, The SSSS samples were artificially aged at $180^{\circ} \mathrm{C}$ in an oil bath. After being taken out of the oil bath at the under-aged stage and quenched, mechanical polishing and buff polishing were immediately conducted, and finishing treatment was carried out by electrolytic polishing. As the electrolyte, a solution mixed at a ratio of perchloric acid: ethylene glycol: ethanol $=1: 2: 7$ was used.

\subsection{Acquisition of GB character distribution}

GB character distribution classified by Brandon's criterion [9] was obtained by electron back scattered diffraction (EBSD) analysis using TSL solution OIM D.C. attached to scanning electron microscopy (FE-SEM), JSM-6500F. Focusing on the rotation angle, the highangle (HAGB), low-angle (LAGB) were selected. In addition, CSL GBs were selected as the special GB. Table 2 summarizes the names of each grain boundary in this study. The step size was selected 10 or $3 \mu \mathrm{m}$. Thereafter, a back scattered electron (BSE) image was obtained by SEM observation, and the microstructure was confirmed.

\subsection{Nanoindentation test}

The relative distance from the indentation position was confirmed from the grain map and the BSE image, and a nanoindentation test (TI950 Tribo indenter) was conducted near the selected GBs. The maximum load was set to $500 \mu \mathrm{N}$, the interval between measurement points was set to 1,2 , or $3 \mu \mathrm{m}$, the loading rate was set to 50 $\mu \mathrm{N} / \mathrm{s}$, and the keeping time was set to $10 \mathrm{~s}$. The indention areas were observed by the BSE image. The distance to the GB was calculated as the vertical distance to the GB.

\subsection{Additional peak-aged heat treatment and other heat treatment}

After the nanoindentation test for $180^{\circ} \mathrm{C}$ and $250^{\circ} \mathrm{C}$ under-aged samples, aging at the same temperature was added to produce a peak-aged samples, and the same GB as the under-aged sample was subjected to the test described in section 2.3. Here, the aging conditions and names of each sample are summarized in Table 3.

\subsection{Microstructure observation}

Ф3 mm disk-shaped samples for transmission electron microscopy (TEM) observation were prepared by twin-jet electrolytic polishing. As the electrolytic solution, a

Table 2

Selected grain boundaries and their misorientation angle.

\begin{tabular}{cccc}
\hline GB & High-angle & Low-angle & $\Sigma 3$ \\
\hline Name & HAGB & LAGB & CSL3 \\
\hline angle $\left[^{\circ}\right]$ & $15 \sim 180$ & $2 \sim 15$ & $60.00 \pm 8.66$ \\
\hline axis & - & - & {$[111]$} \\
\hline
\end{tabular}

Table 3

Aging conditions for each sample.

\begin{tabular}{ccc} 
Name & $180 \mathrm{U}$ & $180 \mathrm{P}$ \\
\hline temperature $\left[{ }^{\circ} \mathrm{C}\right]$ & 180 & 180 \\
\hline time $[\mathrm{h}]$ & 1 & 8 \\
\hline
\end{tabular}


Table 4 Grain boundary character distribution (GBCD) and grain size of $180^{\circ} \mathrm{C}$ aged and SSSS samples.

\begin{tabular}{|c|c|c|c|c|c|c|}
\hline \multirow{2}{*}{ sample } & \multicolumn{5}{|c|}{ GBCD [\%] } & \multirow{2}{*}{ Grain size $[\mu \mathrm{m}]$} \\
\hline & HAGB & LAGB & CSL3 & CSL5 & otherCSL & \\
\hline $180^{\circ} \mathrm{C}$ & 69.1 & 20.2 & 2.02 & 2.85 & 5.11 & $341.8 \pm 136.7$ \\
\hline SSSS & 73.4 & 14.6 & 2.42 & 2.19 & 7.75 & $384.9 \pm 184.0$ \\
\hline
\end{tabular}

solution mixed at a ratio of nitric acid: methanol $=1: 4$ was used. TEM observation (JEM-2000FX) was carried out each sample to observe the microstructures within the grain interior and at the GBs.

\section{Results}

\subsection{Mechanical test}

Table 4 shows the grain boundary character distribution (GBCD) and the grain size of aged sample at $180^{\circ} \mathrm{C}$, an aging heat treatment and a supersaturated solid solution sample prepared for comparison. After aging heat treatment, GBCD and grain size of both samples are almost same. From this comparison, it is considered that the microstructure is not significantly affected by the aging heat treatment.

Fig. 1 shows the nano-hardness of $180^{\circ} \mathrm{C}$ under and peak aged samples. Fig. 1(a) and (d) show the nanohardness around HAGB of under-aged and peak-aged samples, respectively. The average hardness in the grain interior of the peak-aged sample is larger than that of the under-aged sample. The hardness near the GB of the under-aged sample is larger than that of grain interior. In the peak-aged sample, the hardness near the GB is smaller than that of its grain interior. Furthermore, it can be confirmed that the range near the LAGB and the $23 \mathrm{CSL}$ GB where the hardness increases, or decreases is smaller than that near the HAGB.

\subsection{Microstructure}

Fig. 2 shows TEM images of the sample aged at $180^{\circ} \mathrm{C}$. In 180U (Fig. 2(a)), a linear contrast was observed in the matrix, suggesting the precipitation of $\beta^{\prime \prime}$. In 180P (Fig. 2(b)), many needle-shaped contrasts along the $<100>$ direction were uniform in the matrix. And the precipitation of $\beta^{\prime \prime}$ is suggested. Its density is higher than that of the $180 \mathrm{U}$ grain interior.

Precipitates were distributed on the GBs, and large precipitates were sparsely distributed in 180P (Fig. 2(c)) compared to $180 \mathrm{U}$. In addition, the PFZ is formed along the GB under any aging conditions, and the precipitates outside the PFZ are larger than the precipitates in the grain interior. At 180P, it can be confirmed that the precipitates are dispersed at a lower density than in the grain interior.

\section{Discussion}

\subsection{Local deformation behavior around grain boundary}

In $\mathrm{Al}-\mathrm{Mg}-\mathrm{Si}$ based alloys, it is known that the strengthening phase after aging at $180^{\circ} \mathrm{C}$ is $\beta^{\prime \prime}$. Fig. 2 (a) and (b) show that the under-aged sample also contains $\beta^{\prime \prime}$ precipitates that can be confirmed by diffraction contrast. However, the number density is smaller than that of peakaged sample, which means that Mg-Si clusters and GP zones, which are not visible in the diffraction contrast, are

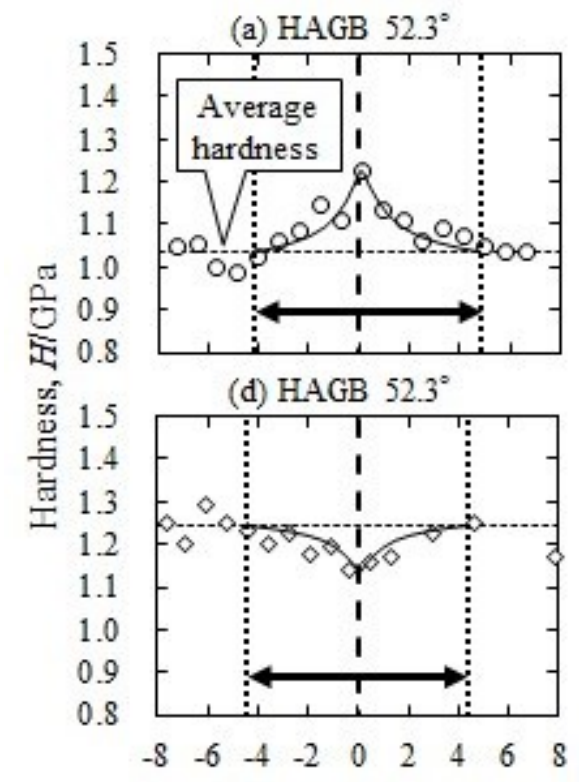

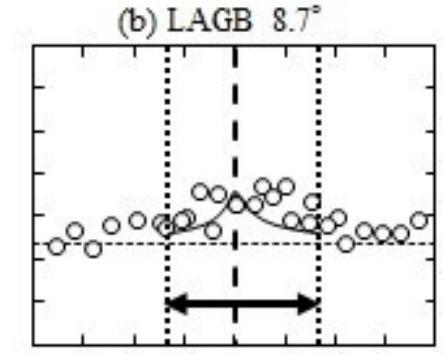

(e) LAGB $8.7^{\circ}$

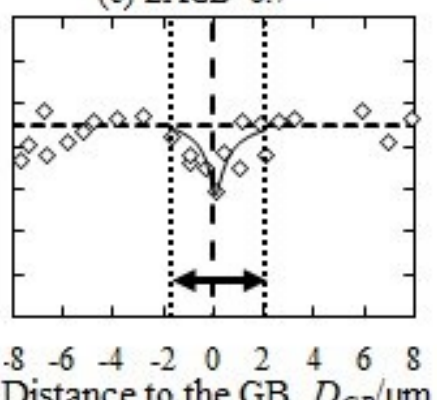

(c) $\operatorname{CSL} 357.3^{\circ}$

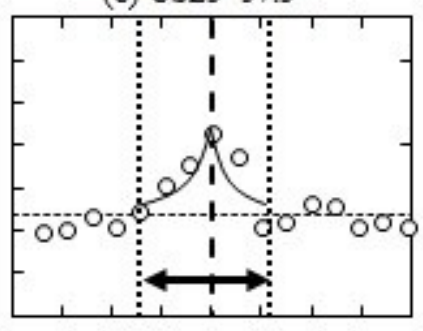

(f) $\operatorname{CSL} 357.3^{\circ}$

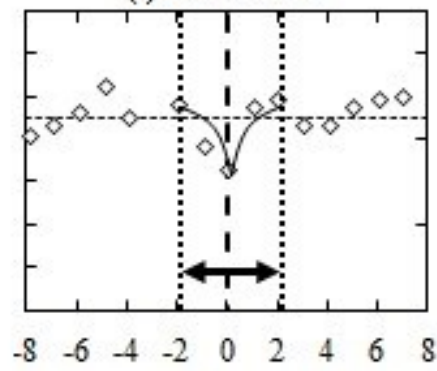

Fig. 1 Nano-hardness around grain boundaries at aging temperature of $180^{\circ} \mathrm{C}$; (a) at under-aged around HAGB, (b) at under-aged around LAGB, (c) at under-aged around CSL3, (d) at peak-aged around HAGB, (e) at peak-aged around LAGB and (f) at peak-aged around CSL3. 
considered to exist densely in the grains. With the increase in the aging time, many $\mathrm{Mg}$-Si clusters and the GP zone become $\beta^{\prime \prime}$ and the number density of $\beta^{\prime \prime}$ increased. Therefore, it can be understood that the average hardness in the grain interior increased at the peak-aged sample than at the under-aged sample.

In Fig. 2 (a) and (c), some larger precipitates than the intragranular precipitates were observed in a region just outside the PFZ. This indicates "Precipitate Sparse Zone: PSZ" and "Transition-area" reported by Ma et al and Ogura et al. [10-11]. In the Al-Zn-Mg based alloy, Ogura et al. reported that during aging heat treatment, the concentrations of $\mathrm{Zn}$ and $\mathrm{Mg}$ dissolved in the matrix decreased as the distance to the GB decreases, and that increasing the aging time also reduced the average $\mathrm{Zn}$ and $\mathrm{Mg}$ concentrations in the PFZ. This indicates that the solute concentration of the matrix shifts to a lower concentration near the GB than in the grain interior, and the solute concentration further decreases by increasing the aging time. Miyazaki et al. [12-13] systematically investigated the change in composition with composition using the "composition gradient method". Miyazaki et al. reported that in $\mathrm{Cu}-\mathrm{Ti}$ and $\mathrm{Ni}-\mathrm{Al}$ alloys, the solute concentration in the matrix decreased as it approached the phase interface, and the precipitates on the lower concentration region were more sparsely distributed than the precipitates on the higher concentration region. This is because there is a dependence between the equilibrium composition at the matrix/precipitate interface and the size of the precipitate, and the precipitation on the low concentration side follows the Gibbs-Thomson equation [14] .

As a result, the critical radius in the precipitation increases, so that the precipitate size increases on the low concentration region. In addition, it is conceivable that precipitates are coarsely distributed by reducing the mass of solute atoms used for precipitation because of decreasing the solute concentration.

Based on the above, "PSZ" confirmed from the results of this experiment is considered to be formed in the following process.

(1) The solute atoms diffuse to the GBs and form GB precipitates.

(2) The solute concentration in the matrix near the GBs decreases due to the formation of GB precipitates.

(3) According to the Gibbs-Thomson relation, large precipitates nucleate at low concentration region.
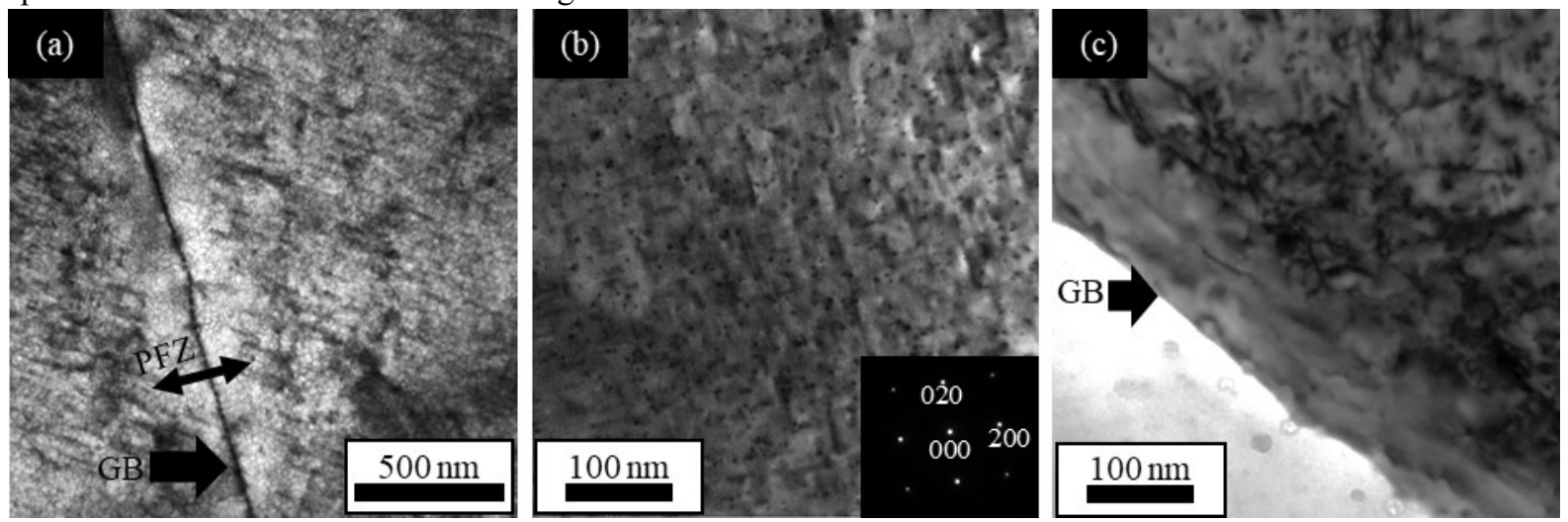

(4) In the low-concentration region, precipitates are coarsely distributed because there are fewer solute atoms in the matrix used for precipitation than in the highconcentration region.

From these consideration, it is considered that larger precipitates were nucleated and coarsely distributed because the concentration near the GBs further decreased with increasing aging time at $180 \mathrm{P}$. Therefore, it is considered that the amount of precipitation strengthening decreased in PSZ near the GB, and the nano-hardness decreased as compared with the nano-hardness in the intragranular region. On the other hand, in the grain interior of $180 \mathrm{U}$, the precipitation was not sufficient due to the short aging time, and the parallelogram-type $\beta^{\prime \prime}$ which is known as the strengthening phase had not been formed. It is considered that the precipitates dominated by the Orowan-type strengthening mechanism were more occupied than in the grain interior, so that in the grain interior, solid solution strengthening and slight precipitation strengthening acted. In PSZ, the amount of solid solution strengthening decreased, but the amount of precipitation strengthening acted greatly. From this assumption, it is considered that the hardness near the GB showed a relatively higher value than the hardness in the grain interior.

In addition, since there is no precipitate in the PFZ, it is considered that only the solid solution strengthening is affected, but no decrease in hardness on the PFZ in 180U was confirmed. This is because the indentation size is much larger than the PFZ width, and even if the indentation just above the PFZ is affected by the precipitates in the GBs, PSZ and grain interior.

\subsection{Effect of grain boundary characters on precipitation behavior}

From the results of $180 \mathrm{U}$ and $180 \mathrm{P}$, the behavior of mechanical properties near the GB was different depending on the GB character.

Cai et al. [4] suggests that low-angle GBs with a misorientation of $11^{\circ}$ or less have the maximum diffusion activation energy of solute atoms among all types of GBs and are random GBs. It is conceivable that the GB diffusion in the LAGB is much more difficult than in the HAGB. In addition, the atomic matching of $\Sigma 3$ is good, so that solute atoms are less likely to diffuse than random

Fig. 2 TEM images of the aged samples, (a): 180U, (b): grain interior in 180P, (c): around GB in 180P. 
GBs. When the GB is regarded as a dislocation array, it is considered that the random GB contains more nucleation sites than the LAGB and low- $\Sigma$ CSL GB. Therefore, the volume fraction of the precipitate on the random GB is larger than that of the LAGB or low- $\Sigma$ CSL GB. As a result, the PFZ width around HAGB also increases.

From the results of nanoindentation on the $180^{\circ} \mathrm{C}$ aged sample, PSZ of the hardness near LAGB and CSL3 is smaller than that of HAGB because the volume fraction of precipitates on GB is small and the PFZ width is narrow. This is probably due to the small influence of the precipitate in PSZ.

From the above results, it is suggested that influence of the GB character on the aging precipitation behavior exists.

Furthermore, in this experiment, the influence of the GB plane normal were not considered, so further investigation is needed in the future.

\section{Conclusion}

Nano-indentation and TEM observation were performed on Al-Mg-Si alloys to investigate how GB characteristics affect aging precipitation behavior and local mechanical behavior. The summary of the results obtained is as follows.

(1) At $180^{\circ} \mathrm{C}$ aging, in the under-aged stage, the density of the precipitates having high resistance increased on the grain boundaries and PSZ, so that the nano-hardness increased as decreasing the distance to the GBs. In the peak-aged stage, large precipitates nucleated due to the decrease in the solute concentration near the GBs. The coarse distribution of the precipitate reduced the amount of precipitation strengthening, and decreased the hardness as approaching the GB.

(2) The volume fraction of precipitates is smaller on LAGB and CSL3 than on HAGBs due to the difficulty of diffusion of solute atoms. Therefore, the PFZ width and the PSZ become narrower, and the range affected by precipitates on the PSZ around LAGB and CSL3 becomes narrower than around HAGB.

\section{References}

1. G. A. Edwards, K. Stiller, G. L. Dunlop, M. J. Couper, Acta Materialia, 46, 11 (1998).

2. M. Murayama, K. Hono, Acta Materialia, 47, 5 (1999).

3. K. Matsuda, J. Tsukiyama, Y. Uetani, S. Ikeno, Journal of Japan Institute of Light Metals, 58, 8 (2008)[in Japanese].

4. B. Cai, B. L. Adams, T. W. Nelson, Acta materialia, 55 (2007).

5. P. N. T. Unwin, G. W. Lorimer and R. B. Nicholson: Acta Met., 17 (1969).

6. K. Saitoh, H. Kitagawa, A. Nakatani and S. Ogata: Journal of the Society of Materials Science, Japan, 46 (1997)[in Japanese].

7. T. Watanabe, Res Mechanica, 11 (1984).

8. M. Shimada, H. Kokawa, Z. J. Wang, Y. S. Sato, I. Karibe, Acta Materialia, 50 (2002).

9. D. G. Brandon, Acta marerialia, 14 (1993).
10. P. P. Ma, C. H. Liu, Q. Y. Chen, Q. Wang, L. H. Zhan, J. J. Li: Journal of Materials Science \& Technology 46 (2020).

11. T. Ogura, S. Hirosawa, T. Sato, Science and Technology of Adovanced Materials, 5 (2004).

12. T. Miyazaki, T. Koyama, and S. Kobayashi, Metallurgical and Materials Transactions, 27A (1996).

13. T. Miyazaki, S. Kobayashi, and T. Koyama, Metallurgical and Materials Transactions, 30A (1999).

14. J. W. Martins and R. D. Doherty: Stability of Microstructure in Metallic System, Cambridge University Press, Cambridge, United Kingdom, 1976 